\title{
An advanced experimental approach for detailed in-situ characterization of interface delamination
}

\author{
J.P.M. Hoefnagels ${ }^{1, a}$, M. Kolluri1 ${ }^{1,2}$, J.A.W. van Dommelen ${ }^{1}$, and M.G.D. Geers ${ }^{1}$ \\ 1 Eindhoven University of Technology, Department of Mechanical Engineering, P.O.Box 513, 5600MB, \\ Eindhoven, The Netherlands. \\ 2 Materials Innovation Institute (M2i), P.O. Box 5008, 2600GA, Delft, The Netherlands.
}

\section{Introduction}

System-In-Package (SIP) microsystems, contain several interfaces formed between stacked, multiple, thin layers, manufactured using different materials and processes. Interface delamination is often recognized as a critical failure mechanism concerning the reliability of these products due to the high stresses at the interfaces. Accurate measurement of interface fracture toughness/ critical energy release rate (CERR) in these miniature interface systems is an ongoing challenge. In addition, modeling of these interfaces requires good understanding of the underlying delamination mechanisms which is even more difficult to obtain with the existing (macroscopic) experimental setups.

\section{Experimental setup}

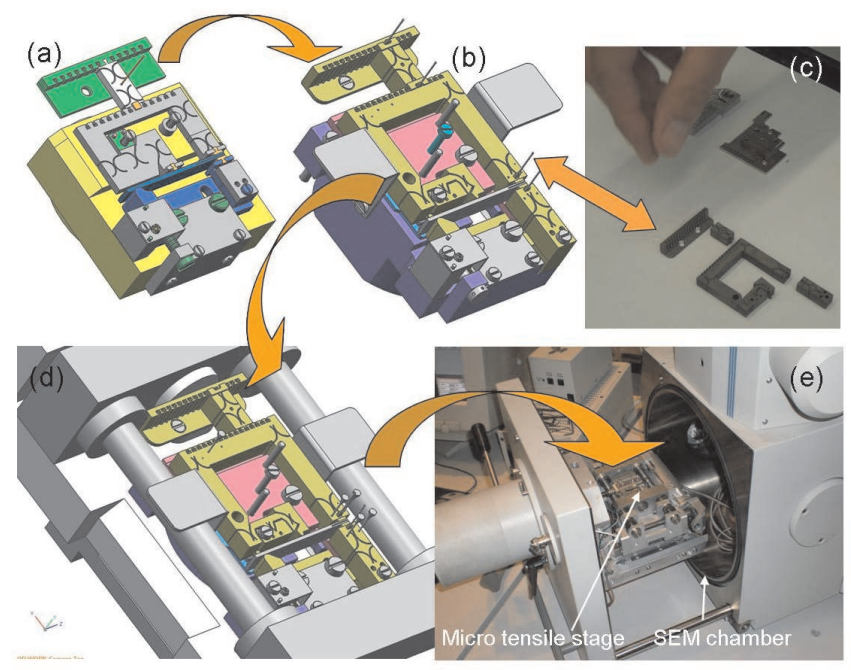

Fig. 1. (a)Previous MMMB setup [1], (b) improved MMMB setup, (c) real picture of the setup illustrating its small size (d) mounted in the micro-tensile stage and (d) eventually in the SEM chamber.

\footnotetext{
${ }^{a}$ e-mail: j.p.m.hoefnagels@tue.nl
} 
In this paper, an improved version of the previously developed miniature mixed mode bending (MMMB) setup [1] capable of in-situ characterization of interface delamination [2] is demonstrated. The functionality of the MMMB setup and its capability to extract intrinsic mechanical interface properties from delamination experiment were proven from tests conducted on custom made interface samples.

\section{Results}

The improved MMMB setup was used to study lead frame - molding compound epoxy interface structures (LF - MCE), which grabbed much attention from the research community as well as industry because of their wide applications and frequent failures during manufacturing and processing of these systems. Results from various experiments conducted at different mode mixities as well as in-situ measurements revealing the interface fracture mechanism during delamination will be discussed.
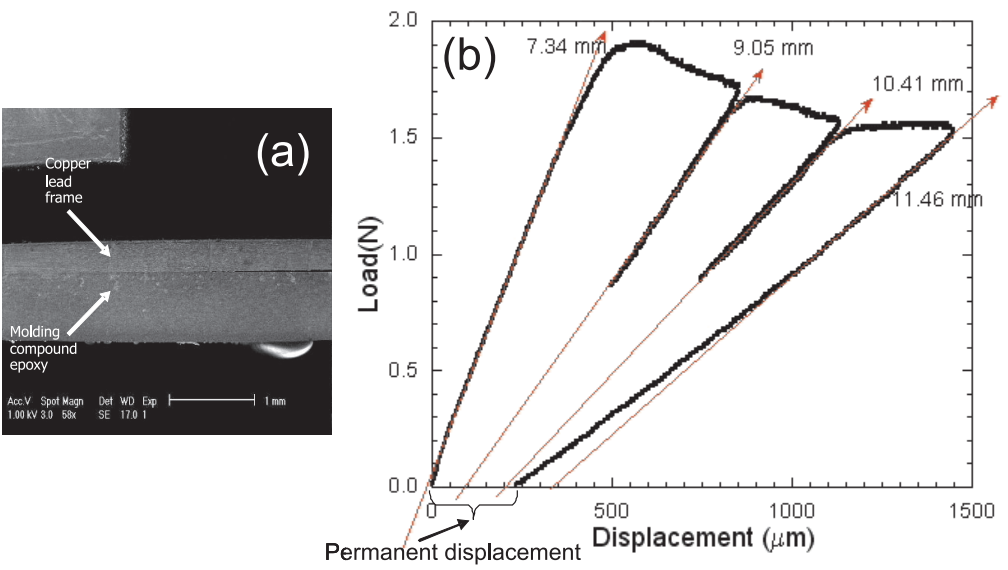

Fig. 2. (a) SEM image of the Cu LF - MCE interface sample, (b) Load displacement response from Mode I delamination test. Permanent deformation is observed after complete unloading.

\section{Analysis and discussion}

An important observation is the presence of permanent deformation after complete unloading, which could originate from bulk and/or interface plasticity. The consequences of this plastic dissipation on the load displacement response and calculation of CERR was studied extensively from a finite element analysis with elasto-plastic material model with an advanced cohesive zone (unloading with damage/ plasticity) interface model. This analysis showed the necessity for detailed microscopic information from the local delamination front to isolate other plastic contributions from interface dissipation. A procedure to identify the relevant microscopic data with the use of digital image correlation on in-situ SEM images of real-time delamination tests with our new experimental will be presented.

\section{References}

1. M. Kolluri, M.H.L. Thissen, J.P.M. Hoefnagels, J.A.W. van Dommelen, M.G.D. Geers, in the Proceedings of EuroSimE 2008 Conference, Freiburg, Germany, (2008) 1.

2. M. Kolluri, M.H.L. Thissen, J.P.M. Hoefnagels, J.A.W. van Dommelen, M.G.D. Geers, Int. J. Frac., 158,(2009) 183. 